\title{
Immune alterations induced by a conditioned aversive stimulus: Evidence for a time-dependent effect
}

\author{
DONALD T. LYSLE and KIMBERLY A. MASLONEK \\ University of North Carolina, Chapel Hill, North Carolina
}

\begin{abstract}
This study investigated the immune alterations induced in rats by an aversive conditioned stimulus (CS), which had been developed through pairings with electric shock. The results showed that the CS induced pronounced alterations of splenic lymphocyte function as indicated by a reduction in the mitogenic responsiveness to the T-cell mitogens concanavalin-A (Con-A) and phytohemagglutinin (PHA) and the B-cell mitogen lipopolysaccharide (LPS), a reduction in interleukin-2 production, and a reduction in natural-killer-cell activity. The magnitude of the immune alterations was dependent on the time interval between the training and testing of the CS. There was little evidence of an immunomodulatory effect of the CS 1 day following training, but the effect developed as the interval between training and test was extended $(3,6$, and 12 days). In contrast, a mitogen-stimulation assay using blood lymphocytes showed a reduction in the mitogenic responsiveness to Con-A and PHA that was comparable across the days following training. These results establish a time-dependent, compartment-specific, CS-induced immunomodulatory effect.
\end{abstract}

It is well documented that physically aversive stimuli can have a pronounced effect on immune function. Presentations of electric shock to rats have been shown to decrease the responsiveness of lymphocytes to stimulation by plant lectins (Keller, Weiss, Schleifer, Miller, \& Stein, 1981, 1983; Lysle, Lyte, Fowler, \& Rabin, 1987), decrease natural-killer-cell activity (Cunnick, Lysle, Armfield, \& Rabin, 1988; Shavit, Lewis, Terman, Gale, \& Liebeskind, 1984), and increase susceptibility to tumor challenge (Lewis, Shavit, Terman, Gale, \& Liebeskind, 1983-1984; Lewis et al., 1983).

There is also evidence that the immune alterations produced by physically aversive stimuli can be modulated by psychological factors. Laudenslager and colleagues (Laudenslager, Ryan, Drugan, Hyson, \& Maier, 1983) have demonstrated that rats permitted to escape electric shocks did not show suppression of lymphocyte reactivity, as compared with yoked subjects receiving the same but inescapable shocks.

Several studies have provided evidence that presentations of an aversive conditioned stimulus (CS) developed through pairings with electric shock can suppress the responsiveness of lymphocytes to $\mathrm{T}$ - and B-cell mitogens, decrease natural-killer-cell activity, and decrease the number of antibody-forming cells (Lysle, Cunnick, Fowler, \& Rabin, 1988; Lysle, Cunnick, Kucinski, Fowler, \& Rabin, 1990; Lysle, Cunnick, \& Maslonek, 1991; Zalcman, Richter, \& Anisman, 1989).

This work was supported by a grant to Donald $\mathrm{T}$. Lysle from the National Institute of Mental Health (MH46284). Address correspondence to Donald T. Lysle, Department of Psychology, Davie Hall, CB No. 3270, University of North Carolina at Chapel Hill, Chapel Hill, NC 27599-3270.
Those conditioned effects were shown to be the result of a learned state induced by the conditioned stimulus and not to the electric shock experience itself, or handling, or exposure to just the conditioned stimulus (Lysle et al., 1990). In further evidence of a conditioned effect, the suppression of lymphocyte responsiveness can be attenuated by extinction and preexposure manipulations (Lysle, Cunnick, Fowler, \& Rabin, 1988). Thus, there is strong evidence that the immunomodulatory effects of a CS are the result of a conditioning process.

However, in contrast to most conditioning paradigms, there is evidence suggesting that the immunomodulatory effects of an aversive CS are dependent upon an elapse of time between training and testing of the CS. Zalcman and colleagues (1989) found that presentation of an aversive CS trained 2 weeks prior to the assessment induced a suppression of splenic plaque-forming cell response, whereas exposure to the same CS trained $72 \mathrm{~h}$ prior to the assessment induced an enhancement of the plaqueforming cell response. The notion that an extended time period between training and testing is important to the expression of CS-induced immunomodulatory effects is suggested by the fact that in studies reporting CS-induced immunosuppression the assessments were conducted at least 6 days following training (Lysle, Cunnick, Fowler, \& Rabin, 1988; Lysle et al., 1990; Lysle et al., 1991; Zalcman et al., 1989). Thus, there is evidence to suggest that the temporal interval between the training and testing of the CS may influence the magnitude, and even the direction, of the immunomodulatory effect, but there are no reported studies directly investigating the effect of time between training and testing of the CS.

The purpose of the present research was to investigate the effect of the time interval between training and test- 
ing on the immune alterations produced by an aversive CS. Specifically, we evaluated the effectiveness of the CS to alter immune function when the time interval between training and testing was manipulated from 1 day to 12 days. We measured the mitogenic responsiveness of lymphocytes from the blood and spleen using both $T$ - and B-cell mitogens. In addition, we assessed the cytotoxic activity of natural-killer cells derived from the spleen. We also evaluated the ability of splenic lymphocytes to produce interleukin-2, a T-cell growth factor produced by activated $\mathrm{T}$-lymphocytes.

\section{Method}

\section{Subjects}

Male rats of the Lewis strain, 65 days old and 250-300 $\mathrm{g}$ in weight, were purchased from the Charles River Laboratories (Raleigh, NC). Upon arrival, the subjects were individually caged in a colony room where a reversed day-night (12-h) cycle was maintained through artificial illumination. The subjects received free access to both food and water throughout the experiment and a 2-week acclimation period prior to the experimental manipulations. All manipulations were initiated $1 \mathrm{~h}$ into the dark phase of the cycle.

\section{Conditioning Apparatus}

Eight rodent chambers (BRS/LVE Model RTC-020) served as the conditioning apparatuses. Timer circuitry to the output of a shock generator and scrambler (BRS/LVE Models SG-903 and SC-902) was used to provide an aversive unconditioned stimulus: a $5.0-\mathrm{sec}$, $1.6-\mathrm{mA}$ footshock. The chambers were individually housed in identical sound-attenuating cubicles.

\section{Conditioning Procedure}

The subjects were randomly assigned to four groups $(n=12)$ that received pairings of the conditioning chamber with footshock, with the training ending $1,3,6$, or 12 days prior to the test day. The pairing of the footshock with the conditioning chamber involved 10 daily presentations of a 5 -sec, $1.6-\mathrm{mA}$ footshock on a 4-min variable-time schedule on each of 2 days. This procedure established the chamber cues as an aversive conditioned stimulus. A prior study showed that exposure to the same conditioning and test procedures with a 12-day interval endowed the CS with immunomodulatory properties. Those properties were the result of a conditioning process, as that effect was not due to the electric shock experience itself, or handling, or exposure to just the conditioned stimulus (Lysle et al., 1990). Following their conditioning, the subjects were kept in their home cages, and then a single test session was administered. For that test session, half of the subjects received a 40-min exposure to the conditioning chamber (without the shock), whereas the other half of the subjects were not exposed to the conditioning chamber but remained in the home cages.

Immediately following the test session, each subject was rapidly sacrificed by cervical dislocation with a clamp. The animal was placed on its back and a mid-abdominal incision was made to expose the abdominal aorta. Blood from all subjects was collected into heparinized syringes through 21-ga needles. The spleen was removed and placed in a polypropylene tube containing $7 \mathrm{ml}$ of RPMI-1640 tissue-culture medium (Gibco) that was supplemented with $10 \mathrm{mM}$ hepes (Gibco), $2 \mathrm{mM}$ glutamine (Gibco), and $50 \mu \mathrm{g}$ gentamicin/ml (Gibco).

\section{Mitogen-Stimulation Assay}

A mitogen-stimulation assay was performed with the splenic leukocytes. The mitogens concanavalin-A (Con-A, Sigma) and phytohemagglutinin (PHA, Burroughs-Wellcome) were used to assess
T-lymphocyte responsiveness, and the mitogen lipopolysaccharide (LPS, Difco) was used to assess B-lymphocyte responsiveness.

A single-cell suspension of each spleen was prepared for each subject by gently pressing the tissue between the ends of sterile frosted microscope slides in supplemented RPMI enriched with $10 \%$ fetal bovine serum (Irvine Scientific). Each subject's splenocytes were counted using a NOVA Celltrak II cell analyzer, and cell suspensions were adjusted to $5 \times 10^{6} / \mathrm{ml}$.

The Con-A, PHA, and LPS were prepared to concentrations of $0,1.0$, and $10.0 \mu \mathrm{g} / \mathrm{ml}$ to provide background, suboptimal, and optimal concentrations of each mitogen. One hundred microliters of the mitogen preparations was added in triplicate to the wells of a 96-well, flat-bottom, microtiter plate (Costar 3595). Then $100 \mu \mathrm{l}$ of the adjusted cell suspension was added to each well and the plates were incubated at $37^{\circ} \mathrm{C}$ in a humidified incubator, with $5 \% \mathrm{CO}_{2}$. The cultures were pulsed with $1 \mu \mathrm{Ci}{ }^{3} \mathrm{H}$-thymidine (specific activity $=6.7 \mathrm{Ci} \mathrm{mM}, \mathrm{ICN}$ ) in $50 \mu \mathrm{l}$ of RPMI-1640 during the last $5 \mathrm{~h}$ of a 48-h incubation. The cultures were harvested onto glass-fiber filter paper using a Skatron harvester. The incorporation of ${ }^{3} \mathrm{H}-$ thymidine was determined with a liquid scintillation counter (Beckman Model LS 1701) and expressed as counts per minute.

A standard whole-blood assay was used to assess the mitogenic responsiveness of blood lymphocytes. The blood was diluted 1:5 with supplemented RPMI-1640, and leukocyte counts were determined using a NOVA Celltrak II cell analyzer. Then $100 \mu \mathrm{l}$ of the diluted blood was added in triplicate to the wells of a 96-well, flatbottom, microtiter plate (Costar 3595) containing $100 \mu \mathrm{l}$ of the prepared Con-A, PHA, or LPS $(0,1.0,10.0$, or $20.0 \mu \mathrm{g} / \mathrm{ml})$. The incubation and harvest conditions were the same as above, except that the total incubation time was extended to $96 \mathrm{~h}$, with the cultures being pulsed during the last $18 \mathrm{~h}$. To account for variations in the number of leukocytes plated, the counts per minute were adjusted to counts/minute/ $10^{5}$ leukocytes.

\section{Natural-Killer-Cell Assay}

A natural-killer-cell assay was performed using splenic leukocytes. A murine T-cell lymphoma, YAC-1, was maintained in supplemented RPMI-1640 plus $10 \%$ FCS in a $\mathrm{CO}_{2}$ incubator. On the day of the assay, YAC-1 targets were prepared by being labeled for $70 \mathrm{~min}$ with $200 \mu \mathrm{Ci}$ of sodium chromate-51 $\left({ }^{31} \mathrm{Cr}\right.$, DupontNew England Nuclear); then the YAC-1 cells were washed three times to remove exogenous ${ }^{51} \mathrm{Cr}$. Splenic leukocytes, prepared as above, were used as effectors and were plated in triplicate at 10 , $5,2.5$, and $1.25 \times 10^{5}$ cells per well of a 96-well plate (Costar 3595). The labeled targets were diluted and plated at $1 \times 10^{4}$ cells per well. Effector:target ratios of 100:1, 50:1, 25:1, and 12.5:1 were obtained. Control wells containing only labeled targets were also plated to determine the spontaneous release and maximum release possible. The plates were incubated for $4 \mathrm{~h}$ at $37^{\circ} \mathrm{C}$ in a humidified $\mathrm{CO}_{2}$ incubator. Immediately prior to harvest, the targets in one set of control wells were lysed with $10 \%$ trichloroacetic acid and the microtiter plates were centrifuged at $500 \mathrm{rpm}$ for $5 \mathrm{~min}$. The supernatants were harvested using a multichannel pipette (Titertek, Flow Laboratories). The amount of ${ }^{51} \mathrm{CR}$ released in the supernatant was counted using an LKB gamma counter (CliniGamma Model 1272).

Lytic units (LU) were calculated using a computer program based on the equations of Pross and Maroun (1984). The percent cytotoxicity at all effector:target ratios was utilized to determine $\mathrm{LU}$. Lytic units were based on the number of leukocytes per $10^{7}$ effectors necessary to lyse $20 \%$ of the targets.

\section{Interleukin-2 Assay}

Interleukin-2 (IL-2) is a T-cell growth factor produced by stimulated T-lymphocytes. To test for the functional ability of T-cells to produce IL-2, $5 \times 10^{6}$ splenocytes in $1 \mathrm{ml}$ of media were incubated with 0 (background) or $5 \mu \mathrm{g} / \mathrm{ml} \mathrm{Con-A}$ for $48 \mathrm{~h}$ in a 12-well microculture plate (Costar 3512). The supernatants were then harvested 
and tested for the presence of IL-2. The supernatants and a positive IL-2 control were serially diluted 1:2 (total of six dilutions) in duplicate in a 96-well plate (Costar 3595) using a multichannel pipette (Titertek). The initial dilution was 1:4. The IL-2-dependent cell line, CTLL-2, was washed to remove any exogenous IL-2 and then was diluted in RPMI-1640 plus $10 \% \mathrm{FCS}$ to $1 \times 10^{3} / \mathrm{ml}$. Then $100 \mu \mathrm{l}$ of the CTLL-2 cell suspension was plated in each well and in 4 control wells containing no IL-2. The plates were incubated for $24 \mathrm{~h}$ at $37^{\circ} \mathrm{C}$ in a humidified atmosphere of $5 \% \mathrm{CO}_{2}$. Each well was pulsed with $1 \mu \mathrm{Ci}$ of ${ }^{3} \mathrm{H}$-thymidine $(50 \mu \mathrm{l})$ for the last $5 \mathrm{~h}$ of the incubation. The cultures were then harvested using a Skatron harvester, and the thymidine incorporation was determined using a scintillation counter. The data were expressed as $1 / 2$-max units, as calculated using a computer program (Lilly Research Laboratories).

\section{Statistical Treatment of Data}

Statistical assessments were made using a computerized program for analysis of variance (ANOVA) (Statistix, NH Analytical Software). A 2-way ANOVA was used to assess the number of leukocytes in the blood and spleen, the mean of the triplicate counts per minute for the different concentrations of Con-A, PHA, and LPS, the lytic units from the natural-killer-cell assay, and the $1 / 2$-max values from the interleukin- 2 assay. In the analysis, the first factor was the four different time intervals between training and testing and the second factor was the type of treatment on the test day, that is, exposure to the conditioning chamber or home cage. Standard polynomial contrasts were performed on all data sets. The level of significance for the $F$ test was set at a probability of 0.05 .

\section{RESULTS}

\section{Mitogen-Stimulation Assay}

Spleen assay. The mitogen-stimulation assay showed comparable effects across all concentrations of Con-A, PHA, and LPS. Figure 1 displays the mean counts per minute for the optimal concentration of Con-A $(1.0 \mu \mathrm{g} / \mathrm{well})$, PHA $(1.0 \mu \mathrm{g} / \mathrm{well})$, and LPS $(1.0 \mu \mathrm{g} /$ well $)$. Analysis of variance of the optimal concentration of Con-A, PHA, and LPS showed significant treatment effects for Con-A $[F(1,40)=32.43, p<.0001]$, PHA $[F(1,40)=46.59$, $p<.0001]$, and LPS $[F(1,40)=72.06, p<.0001]$. This result indicates that exposure to the CS decreased the responsiveness to both $\mathrm{T}$ - and $\mathrm{B}$-cell mitogens. There were also significant effects of the time interval between training and testing for Con- $\mathrm{A}[F(3,40)=3.40, p<.05]$, PHA $[F(3,40)=3.88, p<.05]$, and LPS $[F(3,40)=$ $3.09, p<.05]$. Polynomial contrasts showed a significant linear effect of the time interval between training and testing, indicating a decrease in mitogenic responsiveness with the increase in the time interval for Con- $\mathrm{A}[F(1,40)=$ $7.37, p<.01]$, PHA $[F(1,40)=11.01, p<.01]$, and LPS $[F(1,40)=8.99, p<.01]$. Most importantly, there was a significant interaction between the treatment effect and the time interval between training and testing for Con-A $[F(3,40)=6.00, p<.01]$, PHA $[F(3,40)=3.15$, $p<.05]$, and LPS $[F(3,40)=3.05, p<.05]$. Polynomial contrasts showed a significant linear interaction between the treatment effect and the time interval, indicating that the CS-induced decrease in mitogenic responsiveness became more pronounced with the increase in the time interval for Con-A $[F(1,40)=16.77, p<.001]$,
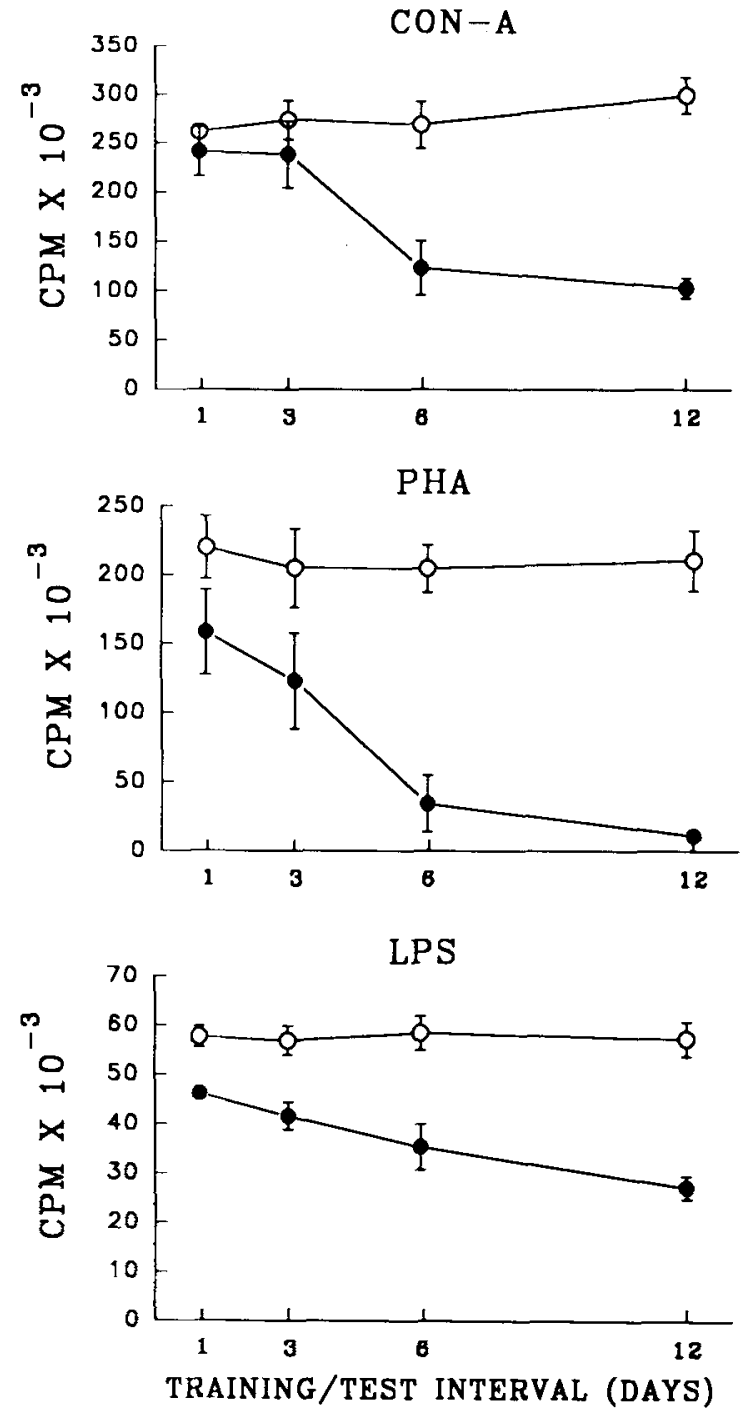

Figure 1. Mitogenic response of splenic lymphocytes induced by the optimal concentration of Con-A, PHA, and LPS, expressed as mean $\pm S E$ of the averaged triplicate counts/minute. The optimal

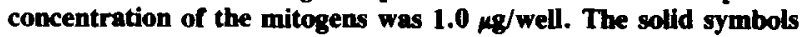
indicate the groups exposed to the conditioned stimulus at different times following training, and the open symbols indicate the groups that remained in their home cages on the test day.

PHA $[F(1,40)=8.87, p<.01]$, and LPS $[F(1,40)=$ $8.99, p<.01]$.

Analysis of variance of the number of spleen leukocytes showed no significant treatment, time interval, or interaction effect. The mean leukocyte count of the spleen was $679.8 \times 10^{6}$ with an $S E M$ of $12.09 \times 10^{6}$.

These results establish that a conditioned aversive stimulus can have a dramatic suppressive effect on the mitogenic responsiveness of splenic T- and B-lymphocytes, but that such an effect is dependent on the time interval between training and testing. 

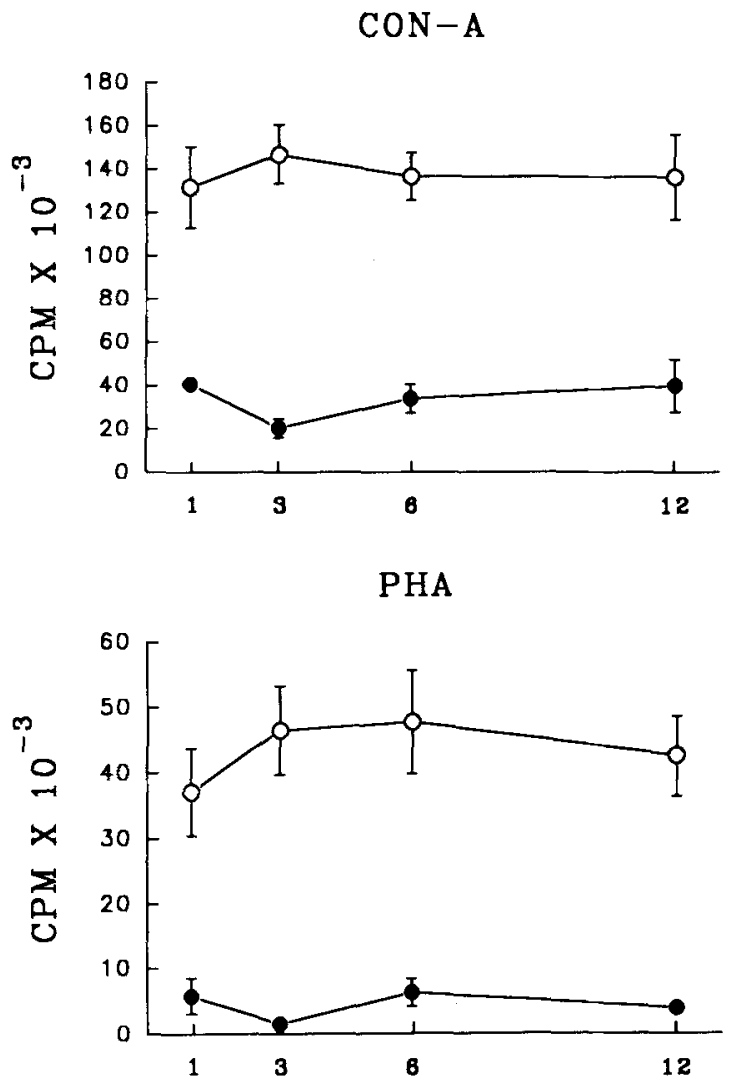

TRAINING/TEST INTERVAL (DAYS)

Figure 2. Mitogenic response of blood lymphocytes induced by the optimal concentration of Con-A and PHA, expressed as mean $\pm S E$ of the averaged triplicate counts/minute/105. The optimal concentration of the mitogens was $1.0 \mu \mathrm{g} /$ well. The solid symbols indicate the groups exposed to the conditioned stimulus at different times following training, and the open symbols indicate the groups that remained in their home cages on the test day.

Whole-blood assay. The results of the whole-blood assay showed comparable effects across all concentrations of Con-A and PHA. LPS did not induce stimulation of the blood lymphocytes above the level of the background wells. Therefore, the results for LPS are not reported. Figure 2 shows the mean counts $/$ minute $/ 10^{5}$ leukocytes for the optimal concentrations of Con-A $(10.0 \mu \mathrm{g} / \mathrm{ml})$ and PHA $(10.0 \mu \mathrm{g} / \mathrm{ml})$. Analysis of variance of the optimal concentrations of Con-A and PHA showed significant treatment effects for Con-A $[F(1,40)=113.97, p<.0001]$ and PHA $[F(3,16)=100.31, p<.0001]$. This result indicates that exposure to the CS decreased the mitogenic responsiveness to both $\mathrm{T}$-cell mitogens. There was no significant effect of the time interval between training and testing and no significant interaction between the treatment and time intervals for Con-A or PHA $(F s<1)$. Thus, in contrast to the results for the splenocytes, the blood lymphocytes showed a reduction in the mitogenic response following exposure to the $\mathrm{CS}$ that was not affected by the time interval between training and testing.
There was also a significant effect of the CS on the number of blood leukocytes. The mean leukocyte count for the subjects exposed to the CS on the test day was $3.54 \times 10^{6} / \mathrm{ml}$ with an SEM of $8.5 \times 10^{5}$, whereas the subjects that remained in their home cages had a leukocyte count of $5.90 \times 10^{6} / \mathrm{ml}$ with an $S E M$ of $1.06 \times 10^{5}$. This finding is consistent with other evidence showing that electric shock can produce a decrease in the number of blood leukocytes without affecting the number of splenocytes (Cunnick, Lysle, Kucinski, \& Rabin, 1990; Keller et al., 1981). This difference between blood and spleen leukocytes further emphasizes the notion that different processes are acting in these different lymphoid compartments.

\section{Natural-Killer-Cell Assay}

The results of the natural-killer-cell assay are displayed in Figure 3. Analysis of lytic units showed a significant treatment effect $[F(1,40)=19.79, p<.0001]$. Although there was no significant main effect of the time interval between training and testing, there was a significant interaction between the treatment and the time interval $[F(3,40)$ $=2.94, p<.05]$. Polynomial contrasts showed a significant linear interaction between the treatment effect and the time interval, indicating that the CS-induced decrease in natural-killer-cell activity became more pronounced with the increase in the time interval $[F(1,40)=8.07$, $p<.01]$. This result indicates that a conditioned aversive stimulus can induce substantial suppression of splenic natural-killer-cell activity, but that such an effect is dependent on the time interval between training and testing.

\section{Interleukin-2 Assay}

The results of the bioassay for IL-2 in the Con-Astimulated samples are displayed in Figure 4. Analysis of $1 / 2$-max units showed a significant treatment effect

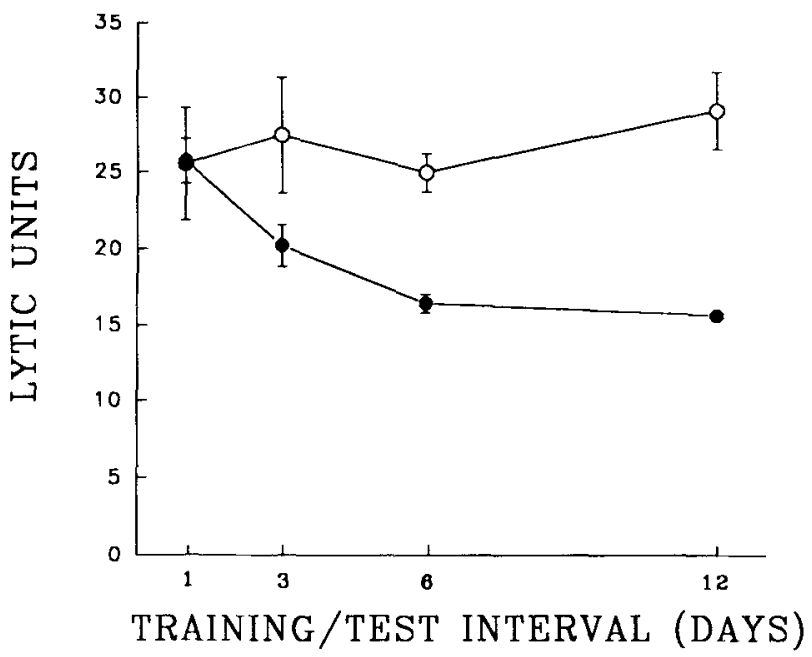

Figure 3. Splenic natural-killer-cell activity expressed as lytic units (mean $\pm S E$ ). The solid symbols indicate the groups exposed to the conditioned stimulus at different times following training, and the open symbols indicate the groups that remained in their home cages on the test day. 


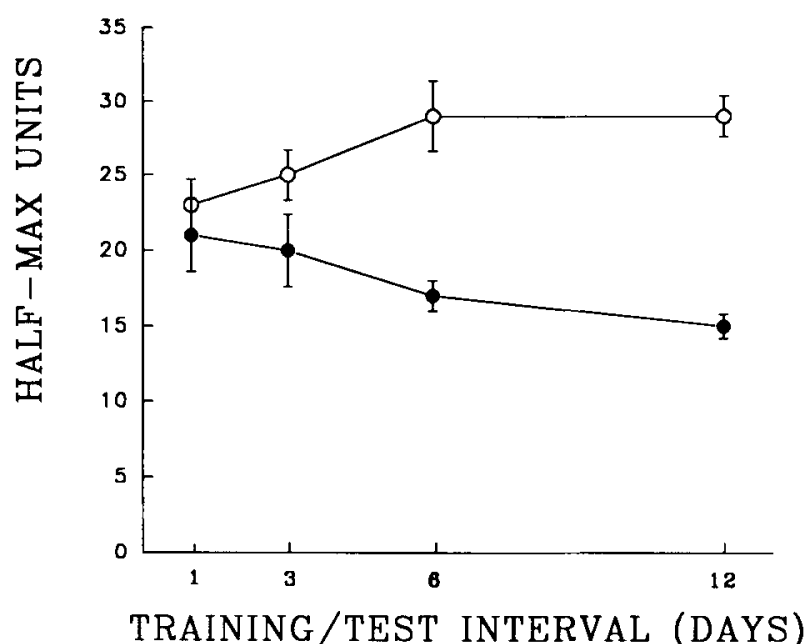

Figure 4. IL-2 production by splenic lymphocytes expressed as $1 / 2$-max values (mean $\pm S E$ ). The solid symbols indicate the groups exposed to the conditioned stimulus at different times following training, and the open symbols indicate the groups that remained in their home cages on the test day.

$[F(1,40)=34.80, p<.0001]$. Although there was no significant main effect of time interval, there was a significant interaction between the treatment and the time interval $[F(3,40)=3.91, p<.05]$. Polynomial contrasts showed a significant linear interaction between the treatment effect and the time interval, indicating that the CSinduced decrease in IL-2 production became more pronounced with the increase in the time interval $[F(1,40)=$ $11.54, p<.01]$. The bioassay did not show any detectable IL-2 in the background samples. These results indicate that an aversive CS can induce a substantial decrease in IL-2 production by splenic lymphocytes, but, as with the other measures of splenic immune function, such an effect was dependent on the time interval between training and testing.

\section{DISCUSSION}

In this study, exposure to an aversive CS induced pronounced alterations of splenic lymphocyte function as indicated by a reduction in the mitogenic responsiveness to Con-A, PHA, and LPS, a reduction in interleukin-2 production by Con-A stimulation, and a reduction in naturalkiller-cell activity. The magnitude of these immune alterations was positively related to the time interval between the training and testing of the CS. There was little evidence of an immunomodulatory effect of the CS 1 day following training, but that effect developed over the next several days $(3,6$, and 12 days). In contrast, the CS induced a reduction in the total number of blood leukocytes and a reduction in the mitogenic responsiveness to Con- $A$ and PHA that was comparable across the days following training. Collectively, these results establish that the immunomodulatory effect of the CS is dependent on the time interval between training and testing as well as on the particular compartment of the immune system assessed.

The present results are consistent with other work that has shown that unconditioned aversive stimuli such as electric shock and 2-deoxy-D-glucose (an antimetabolic glucose analogue that produces an acute intracellular glucoprivation) have differential effects on immune compartments (Lysle, Cunnick, Wu, et al., 1988; Lysle et al., 1987). Those studies showed that while both splenic and blood lymphocytes are suppressed with the initial presentations of electric shock or with the first injection of 2-deoxy-D-glucose, the suppression for the spleen lymphocytes but not the blood lymphocytes attenuates with continued presentation of those stimuli.

Taken together, these studies suggest that the lack of CS effectiveness immediately following training could be the result of a habituation effect developed during training. Moreover, such an interpretation would postulate that with time the habituation effect dissipates, allowing the CS to induce a reduction in immunoresponsiveness. The finding of a habituation effect of spleen lymphocytes but not blood lymphocytes is consistent with the present findings of a time-dependent effect for splenic but not blood lymphocytes. However, this interpretation has difficulty accounting for the time-dependent effect of the CS on natural-killer-cell activity, since the suppression of naturalkiller-cell activity by electric shock does not attenuate with repeated exposure to the shock (Cunnick et al., 1988). Furthermore, such an interpretation cannot easily account for the CS-induced enhancement of the plaque-forming cell response observed by Zalcman and colleagues (Zalcman et al., 1989).

The present study demonstrates a time-dependent change in the effectiveness of the CS to induce immunomodulation, but the mechanism responsible for this effect has not been determined. In a recent study from our laboratory, it was shown that the administration of propranolol, a $\beta$-adrenergic receptor antagonist, completely blocked the suppression of splenic mitogenic responsiveness induced by an aversive CS (Lysle et al., 1991). This finding suggests that a time-dependent change in the release of catecholamines or in the density of the $\beta$-adrenergic receptors may be responsible for the time-dependent changes in the effectiveness of the CS.

Regardless of the mechanism responsible for the timedependent effect, the results of the present study are important because they demonstrate that a sufficient time interval between training and testing of the CS may be necessary for observation of CS-induced immune alterations of splenic lymphocytes.

\section{REFERENCES}

Cunnick, J. E., Lysle, D. T., Armfield, A., \& Rabin, B. S. (1988). Shock-induced modulation of lymphocyte responsiveness and natural killer activity: Differential mechanisms of induction. Brain, Behavior, \& Immunity, 2, 102-113.

Cunnick, J. E., Lysle, D. T., Kucinski, B. J., Rabin, B. S. (1990). Evidence that shock-induced immune suppression is mediated by 
adrenal hormone and peripheral beta-adrenergic receptors. Pharmacology, Biochemistry \& Behavior, 36, 645-651.

Keller, S. E., Weiss, J. M., Schleifer, S. J., Miller, N. E., \& STEIN, M. (1981). Suppression of immunity by stress: Effect of a graded series of stressors on lymphocyte stimulation in the rat. Science, $213,1397-1400$.

Keller, S. E., Weiss, J. M., Schleifer, S. J., Miller, N. E., \& STEIN, M. (1983). Stress-induced suppression of immunity in adrenalectomized rats. Science, 221, 1301-1304.

Laudenslager, M. L., Ryan, S. M., Drugan, R. C., Hyson, R. L., \& MAIER, S. F. (1983). Coping and immunosuppression: Inescapable but not escapable shock suppresses lymphocyte proliferation. Science, 221, 568-570.

Lewis, J. W., Shavtr, Y., Terman, G. W., Gale, R. P., \& Liebeskind, J. C. (1983-1984). Stress and morphine affect survival of rats challenged with a mammary ascites tumor (MAT 13762B). Natural Immunity \& Cell Growth Regulation, 3, 43-50.

Lewis, J. W., Shavit, Y., Terman, G. W., Nelson, L. R., Gale, R. P., LIEBEskind, J. C. (1983). Apparent involvement of opioid peptides in stress-induced enhancement of tumor growth. Peptides, 4, 635-638.

Lysle, D. T., Cunnick, J. E., Fowler, H., \& Rabin, B. S. (1988). Pavlovian conditioning of shock-induced suppression of lymphocyte reactivity: Acquisition, extinction, and preexposure effects. Life Sciences, 42, 2185-2194.

Lysle, D. T., Cunnick, J. E., Kucinski, B. J., Fowler, H., \&
RABIN, B. S. (1990). Characterization of immune alterations induced by a conditioned aversive stimulus. Psychobiology, 18, 220-226.

Lysle, D. T., Cunnick, J. E., \& Maslonek, K. A. (1991). Pharmacological manipulation of immune alterations induced by a conditioned aversive stimulus: Evidence for a $\beta$-adrenergic receptor mediated Pavlovian conditioning process. Behavioral Neuroscience, 105, 443-449.

Lysle, D. T., Cunnick, J. E., Wu, R., Caggiula, A. R., Wood, P. G., \& RABIN, B. S. (1988). 2-deoxy-D-glucose modulation of T-lymphocyte reactivity: Differential effects on lymphoid compartments. Brain, Behavior, \& Immunity, 2, 212-221.

LySLE, D. T., LyTE, M., Fowler, H., \& RABIN, B. S. (1987). Shockinduced modulation of lymphocyte reactivity: Suppression, habituation, and recovery. Life Sciences, 41, 1805-1814.

Pross, H. F., Maroun, J. A. (1984). The standardization of NK cell assays for use in studies of biological response modifiers. Journal of Immunological Methods, 68, 235-249.

Shavit, Y., Lewis, J. W., Terman, G. W., Gale, R. P., \& Liebeskind, J. C. (1984). Opioid peptides mediate the suppressive effect of stress on natural killer cell cytotoxicity. Science, 223, 188-190.

Zalcman, S., Richter, M., ANisman, H. (1989). Alterations of immune functioning following exposure to stressor-related cues. Brain, Behavior, \& Immunity, 3, 99-109.

(Manuscript received April 5, 1991; revision accepted for publication June 20,1991 .) 